\title{
Multi-scale spectrally resolved quantitative fluorescence imaging system: towards neurosurgical guidance in glioma resection
}

Yijing Xie, Maria Thom, Anna Miserocchi, Andrew McEvoy, Adrien Desjardins, et al.

Yijing Xie, Maria Thom, Anna Miserocchi, Andrew W. McEvoy, Adrien Desjardins, Sebastien Ourselin, Tom Vercauteren, "Multi-scale spectrally resolved quantitative fluorescence imaging system: towards neurosurgical guidance in glioma resection," Proc. SPIE 10050, Clinical and Translational Neurophotonics, 1005002 (27 February 2017); doi: 10.1117/12.2250159 


\title{
Multi-Scale Spectrally-Resolved Quantitative Fluorescence Imaging System: Towards Neurosurgical Guidance in Glioma Resection
}

\author{
Yijing Xie ${ }^{\mathrm{a},{ }^{*}}$, Maria Thom ${ }^{\mathrm{b}}$, Anna Miserocchi ${ }^{\mathrm{c}}$, Andrew W. McEvoy ${ }^{\mathrm{c}}$, Adrien Desjardins ${ }^{\mathrm{d}}$, \\ Sebastien Ourselin ${ }^{\mathrm{a}}$, and Tom Vercauteren ${ }^{\mathrm{a},{ }^{*}}$

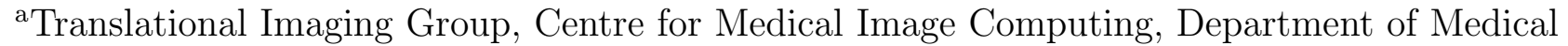 \\ Physics and Biomedical Engineering, University College London, London, UK \\ ${ }^{b}$ Department of Neuropathology, Institute of Neurology, University College London, London, \\ $\mathrm{UK}$ \\ 'Institute of Neurology, National Hospital for Neurology and Neurosurgery, University College \\ London, London, UK \\ ${ }^{d}$ Photoacoustic Imaging Group, Department of Medical Physics and Biomedical Engineering, \\ University College London, London, UK
}

\begin{abstract}
In glioma resection surgery, the detection of tumour is often guided by using intraoperative fluorescence imaging notably with 5-ALA-PpIX, providing fluorescent contrast between normal brain tissue and the gliomas tissue to achieve improved tumour delineation and prolonged patient survival compared with the conventional white-light guided resection. However, the commercially available fluorescence imaging system relies on surgeon's eyes to visualise and distinguish the fluorescence signals, which unfortunately makes the resection subjective. In this study, we developed a novel multi-scale spectrally-resolved fluorescence imaging system and a computational model for quantification of PpIX concentration. The system consisted of a wide-field spectrally-resolved quantitative imaging device and a fluorescence endomicroscopic imaging system enabling optical biopsy. Ex vivo animal tissue experiments as well as human tumour sample studies demonstrated that the system was capable of specifically detecting the PpIX fluorescent signal and estimate the true concentration of PpIX in brain specimen.
\end{abstract}

Keywords: Glioma resection, 5-ALA-PpIX, quantitative fluorescence imaging, fluorescence confocal laser endomicroscope

\section{INTRODUCTION}

Primary malignant cerebral glioma classified as high-grade glioma (HGG) is locally invasive, it can spread to and damage other parts of the brain as well as the spinal cord. It is identified as the most common type of primary cerebral tumour, and the most fatal. Despite the aggressive cyto-reductive treatment, its prognosis is poor with the overall median survival time of approximately 12 months irrespective of age, and with less than $5 \%$ of patients surviving longer than five years. ${ }^{1}$ The goal of the glioma neurosurgery is to achieve a gross-total resection (GTR) meaning that the surgeon has to precisely remove all visibly identified tumours, and no apparent tumours can be determined on the post-operative MRI scans as well as in histopathology. Clinical studies have demonstrated patients who received GTR showed a significantly longer survival than those who had an subtotal resection (STR). ${ }^{1,2}$ For low-grade glioma patients particularly, the five-year overall survival was markedly increased from 50-70\% in STRs to 80-95\% in GTR. ${ }^{1}$ Unfortunately, the average fraction of GTR among all resection surgeries is less than $20 \%$, meaning then patients still have tumour cells remaining in the margins after surgery.

Further author information: (Send correspondence to Yijing Xie)

Yijing Xie: E-mail: y.xie@ucl.ac.uk

Tom Vercauteren: E-mail: t.vercauteren@ucl.ac.uk

Clinical and Translational Neurophotonics, edited by Steen J. Madsen, Victor X. D. Yang, Proc. of SPIE

Vol. 10050, 1005002 - (c) The Authors. Published under a Creative Commons Attribution

CC-BY 3.0 License doi: 10.1117/12.2250159

Proc. of SPIE Vol. 10050 1005002-1 
Emerging intraoperative techniques have been developed and utilised in this scenery to achieve a better determination of the tumour margin, including intraoperative MRI, intraoperative neuronavigation, and as well as intraoperative fluorescence imaging guidance. ${ }^{3,4}$ Among these, the intraoperative 5-ALA-PpIX fluorescence imaging is considered to have a higher sensitivity and specificity for high grade glioma resection compared to iMRI. The oral 5-ALA drug is preferably metabolised into fluorescence protoporphyrin IX (PpIX) molecules by glioma cells, with a ratio around 30:1 between HGGs and normal brain tissue providing fluorescent contrast. ${ }^{5}$ Clinical studies have shown the 5-ALA-PpIX guided excision of high-grade gliomas (HGG) was capable of achieving improved complete resection rates with more than $50 \%$ GTR, and prolonged patient survival compared with the conventional white-light guided resection. ${ }^{6-8}$ However, 5-ALA-PpIX emits very weak fluorescence in low-grade gliomas (LGG), at the infiltrated margins of HGG, and occasionally in non-malignant regions, subsequentially obscuring the tumour margins. ${ }^{6}$ Furthermore, current commercially-available fluorescence imaging systems rely on surgeon's eyes to visualise and distinguish the fluorescence signals, which unfortunately makes the resection subjective. As such, there is an urgent need to develop a spectrally-resolved quantitative fluorescence imaging system to facilitate reliable and maximised tumour resection by providing sufficient specificity and sensitivity on the fluorescence detection. Some research groups have reported their approaches on quantitative assessment of 5-ALA-PpIX fluorescence for glioma resection by using fibre-optic spectroscopy measurements. ${ }^{9-11}$ Among them, Valdés and colleagues developed an intraoperative contact optical probe and a mathematic model to extract the absolute concentration of the fluorophore PpIX in the examined tissue, providing neurosurgeons a brief estimation of the tumour pathological degree. ${ }^{12}$ However, since the probe-based quantification method only determined the local concentration of PpIX in brain tissue, it remains challenging to implement the quantification for the whole surgical area.

In this work, we introduced a multi-scale spectrally-resolved quantitative fluorescence imaging modality aiming to provide an effective computer-assisted surgical guidance for glioma resection. It included a widefield quantitative spectrally-resolved imaging system to provide quantitative PpIX concentration map over the entire wide-field image, and a fibre-based laser confocal endomicroscope to facilitate in situ optical biopsy. We elaborated a computational model based on light propagation theory and least square estimation to calculate the absolute value of the PpIX concentration. We demonstrated our approach was capable of quantitative estimation of fluorescence signal from PpIX in excised brain tissue.

\section{MATERIALS AND METHODS}

\subsection{Imaging System Setup}

\subsubsection{Wide-Field Spectrally-Resolved Quantitative Fluorescence Imaging System (WFQI)}

The WFQI system developed in this study consists of three main modules, 1) light source, which includes a white light LED (MCWHF1, Thorlabs) and a $405 \mathrm{~nm}$ LED (M405FP1, Thorlabs), 2) a liquid crystal tunable filter (LCTF) (VariSpec-VIS, PerkinElmer), 3) and a high performance CMOS camera (ORCA-Flash4.0, Hamamatsu Photonics) (shown in Fig. 1(a)). The $405 \mathrm{~nm}$ LED is chosen specifically for PpIX excitation, as the peak excitation wavelength of PpIX locates at around $405 \mathrm{~nm}$ (Fig. 1(b)). The LCTF performs as a band-pass filter that controllably selects a single wavelength (with $20 \mathrm{~nm}$ full-width at half-maximum) of light to transmit into the camera with a maximum transmittance of $55 \%$ at $710 \mathrm{~nm}$. It features a spectral range from $400-720 \mathrm{~nm}$ with a tuning resolution of $5 \mathrm{~nm}$ and a response time of $50 \mathrm{~ms}$. The high sensitive camera used has $2048 \times 2048$ pixels; each pixel size is $6.5 \mu m \times 6.5 \mu m$. The wavelength-specific images are acquired by using custom LabView (National Instruments, Austin, TX) program, which implements hardware control, data acquisition, data storage and data processing.

\subsubsection{Probe-based Fluorescence Confocal Laser Endomicroscope (pCLE)}

PCLE (Cellvizio, Mauna Kea Technologies) is capable of obtaining in situ high resolution microscopic images as it utilises a flexible miniaturised optical probe ${ }^{14}$ (Fig. 1(a)). The optical fibre bundle probe consisting of 30,000 fibres, yielding a field of view (FoV) of $240 \mu \mathrm{m} \times 240 \mu \mathrm{m}$. A dual-axes scanning unit is integrated to create a raster scanning pattern through each fibre's core in the fibre bundle, giving a scanning rate of $12 \mathrm{fps}$. The system includes an embedded laser source with centre wavelength at $488 \mathrm{~nm}$. Although this wavelength does not fall into the peak excitation range of PpIX, it is able to provoke PpIX fluorescence by leveraging its unique feature of multiple excitation wavelengths (Fig. 1(b)). 


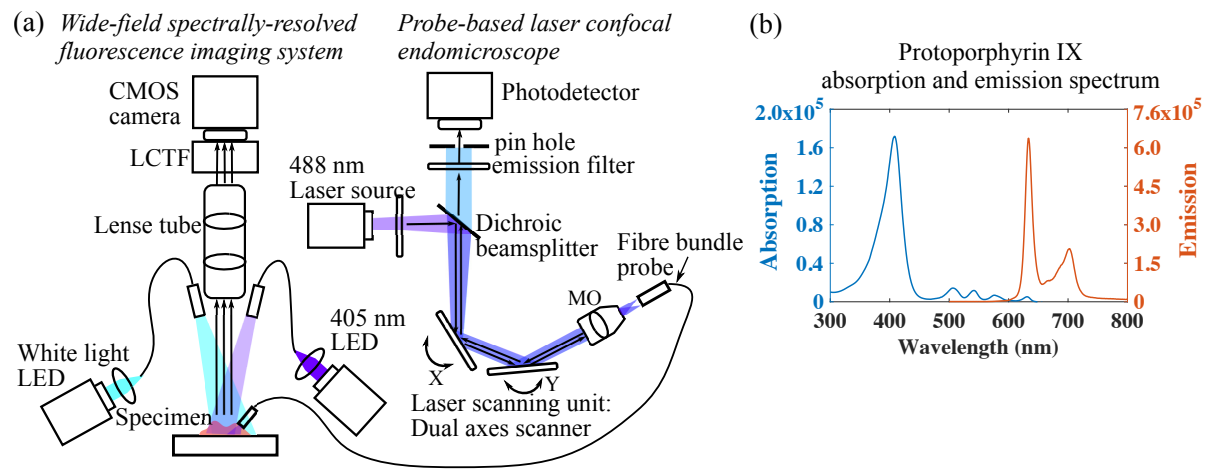

Figure 1. (a) Schematic of system setup. (b) The excitation and emission spectrum of PpIX (A.U.). ${ }^{13}$ LCTF: liquid crystal tunable filter, MO: microscope objective.

\subsection{Data Acquisition and Quantification Algorithms}

The fluorescence excitation was provided by using the fibre-coupled $405 \mathrm{~nm}$ LED through a beam collimator, whereas the diffuse reflectance excitation was implemented using the fibre-coupled white light LED with diverged beam. The power density of $405 \mathrm{~nm}$ light and white light at the sample surface were measured as $1.45 \mathrm{~mW} / \mathrm{cm}^{2}$ and $0.1 \mathrm{~mW} / \mathrm{cm}^{2}$, respectively. For both excitation scheme, 3D image cubes $(x, y, \lambda)$ were produced from successive CMOS camera frames when scanning the tuning wavelength of the LCTF across its full spectral range (400-720 nm) with $10 \mathrm{~nm}$ incrementing steps, in a manner of one frame per tuning wavelength. The exposure time was set to $0.1 \mathrm{~s}$ for all wavelengths under both excitation scenarios to achieve a sufficient signal-to-noise ratio (SNR). $2 \times 2$ pixel binning was utilised to further enhance SNR, which generated $1024 \times 1024$ pixels images. In this way, each image cube contained $(x) 1024 \times(y) 1024 \times(\lambda) 33$ spectra-specific pixels. Especially, for each pixel $(i, j)$ in an image, the fluorescence spectrum responses using $405 \mathrm{~nm}$ excitation $f_{i, j}^{f l u o}(\lambda)$ and diffuse reflectance spectrum using white light excitation $f_{i, j}^{\text {diff }}(\lambda)$ were constructed.

In biological tissue, the fluorescence spectrum is usually modulated when using $405 \mathrm{~nm}$ excitation light, because the absorption of hemoglobin is notably greater in the blue-green region $(380-480 \mathrm{~nm})$ than the red region in its absorption spectrum. ${ }^{15}$ Furthermore, the optical scattering property of biological tissue also affects the spectra for both fluorescence and diffuse reflectance with respect to path lengths of the collected photons. As such, the measured fluorescence spectra from PpIX in biological tissue is a modulated spectra, which does not trivially reflect the true concentration of the PpIX. We applied a computational model to eliminate the influences from tissue's optical properties. The model is based on the light propagation theory ${ }^{12}$ assumption that most of the light attenuation is due to absorption at the excitation wavelength, and scattering at the emission band. A brief flow chart of the computation procedure is demonstrated in a schematic diagram in Fig. 2. For each pixel,

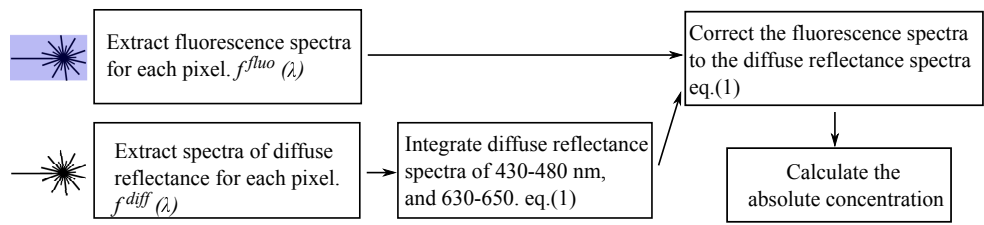

Figure 2. Flow chart of the data processing procedure.

the fluorescence spectra $f_{i, j}^{f l u o}(\lambda)$ and diffuse reflectance spectra $f_{i, j}^{d i f f}(\lambda)$ were extracted from the image cube $(x, y, \lambda)$. The measured fluorescence spectra is normalised by the following equation

$$
\begin{gathered}
f_{(i, j) \text { nor }}^{\text {fluo }}(\lambda)=\frac{f_{\text {measured }}^{\text {fluo }}(\lambda)}{F_{\text {ex }}^{\text {diff }} \times\left(F_{\text {em }}^{\text {diff }}\right)^{\alpha}} \\
F_{\text {ex }}^{\text {diff }}=\int_{430}^{480} f_{i, j}^{\text {diff }}(\lambda) \mathrm{d} \lambda, \quad F_{\text {em }}^{\text {diff }}=\int_{630}^{650} f_{i, j}^{\text {diff }}(\lambda) \mathrm{d} \lambda
\end{gathered}
$$


where $F_{e x}^{d i f f}$ and $F_{e m}^{\text {diff }}$ are the reflectance signals integrated over the wavelength ranges $430-480 \mathrm{~nm}$ (excitation) and $630-650 \mathrm{~nm}$ (emission), respectively. The scattering effect at the emission band can be corrected with an empirical power function $\alpha$ due to the fluorescence efficiency $(\alpha=-0.7) \cdot{ }^{16}$ A least-squares estimate was applied to the corrected fluorescence spectra to calculate the true PpIX concentration at the coordinate $x_{i}, y_{j}$ of the image, using the spectra of a phantom $(R(\lambda))$ with known unit PpIX concentration $(1 \mu \mathrm{g} / \mathrm{ml})$ as the reference through

$$
C_{i, j}^{\text {true }}=\left(R(\lambda)^{T} R(\lambda)\right)^{-1} R(\lambda)^{T} f_{(i, j) \text { nor }}^{\text {fluo }}
$$

\subsection{Ex vivo Animal Studies}

Freshly excised lamb brains were collected from a local butcher shop. The brain tissue was sliced into approximately $2 \mathrm{~cm} \times 2 \mathrm{~cm} \times 0.5 \mathrm{~cm}$ block. Then the brain block was immersed into $100 \mu \mathrm{g} / \mathrm{ml}$ the fluorescent medium Protoporphyrin IX disodium salt (Sigma, UK) for $15 \mathrm{~min}$, then rinsed thoroughly with PBS (Phosphate-buffered saline). The reason of using PpIX instead of 5-ALA which is applied in the clinical practices is the ex vivo brain specimen is no longer capable of metabolising 5-ALA and synthesising PpIX. All specimens were kept in dark to avoid $405 \mathrm{~nm}$ excitation from the ambient light prior to imaging.

\subsection{Excised Human Glioma Studies}

The study was approved by the Joint Research Ethics Committee of the National Hospital for Neurology and Neurosurgery, the UCL Institute of Neurology, and Epilepsy Society Brain and Tissue Bank. Tumour specimens were collected from patients undergoing glioma resection surgery after obtaining consent, and all the experimental procedures were in accordance with the current UK Human Tissue Authority guidelines. The specimen used in this study was selected and sliced from the original excised tumour tissue by neuropathologist. For the cases where patients were not preoperatively administered 5-ALA, the specimens received topically application of PpIX using the same procedure described previously. Right after the imaging experiment, the specimens were returned to the neuropathology lab for histopathology.

\section{RESULTS AND DISCUSSIONS}

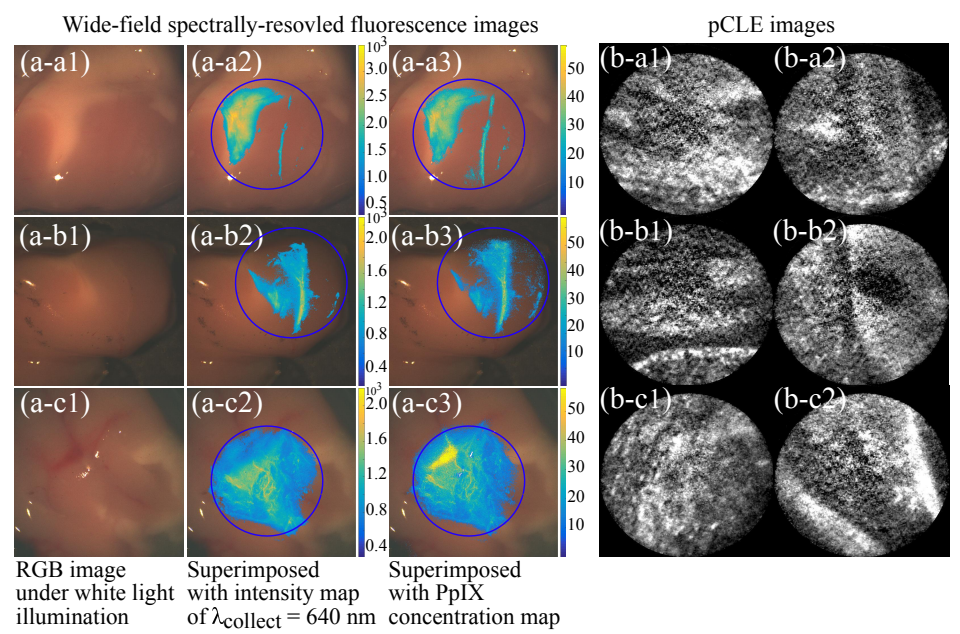

Figure 3. (a) Wide-field fluorescence images and estimations of $C_{P p I X}$ in animal brain samples. (a-a1,b1,c1) White light FoV images. (a-a2,b2,c2) Corresponding fluorescence images demonstrating PpIX fluorescence at $\lambda=640 \mathrm{~nm}$. (a-a3,b3,c3) Map of estimation of concentration of PpIX displaying $C_{P p I X}>10 \mu \mathrm{g} / \mathrm{ml}$. (b) Fluorescence endomicroscopic images of the corresponding samples. The blue circles indicate the UV-illuminated area.

The system and the proposed computational model was first validated with phantom experiments, where optical phantoms that resembled brain tissue's optical properties and with known PpIX concentration were developed. The estimated PpIX concentrations presented good correspondence with the true concentration. Ex vivo Animal Studies Four lamb brains were examined in this study using the wide-field quantitative 
spectrally-resolved fluorescence imaging device and the fluorescence confocal laser endomicroscope. The wide field white light RGB images were composed from superimposing three spectra images in respect of red 630 $\mathrm{nm}$, green $540 \mathrm{~nm}$ and blue $470 \mathrm{~nm}$. We constructed spectra-specific intensity map of $\lambda=640 \mathrm{~nm}$ which is the peak emission under UV excitation to view the PpIX distribution in the UV-illuminated area of the brain tissue. We used the quantification method introduced in this study to construct a true PpIX concentration map over the corresponding area. Fig. 3 shows three representative samples with RGB images of the specimen, the superimposed images, as well as the corresponding endomicroscopic images. We found that notably high intensity signal at $\lambda=640 \mathrm{~nm}$ presented in the white matter and blood vessels (Fig. 3.a2-c2). Presumably, the fibrous structures in the white matter such as axon bundle or neural fibre facilitated the diffusion of PpIX into the tissue. Likewise, blood vessel in the specimen probably also played a role in transportation of the PpIX solution. Maps of true PpIX concentrations displaying $C_{P p I X}>10 \mu \mathrm{g} / \mathrm{ml}$ were superimposed with the corresponding anatomic images, shown in Fig. 3.a3-c3. Noticeably, in specimen c a vessel structure of which the determined true $C_{P p I X}$ was markedly higher compared to the intensity of spectra of $\lambda=640 \mathrm{~nm}$ in the corresponding area. The $C_{P p I X}$ in this hemoglobin-rich structure was corrected and enhanced by the proposed algorithm, since the high absorption in hemoglobin at PpIX excitation wavelength was regulated by using the correction method. While the anatomic pattern revealed in the corresponding fluorescence endomicroscopic images were presumably from the fibrous structure and blood vessels.

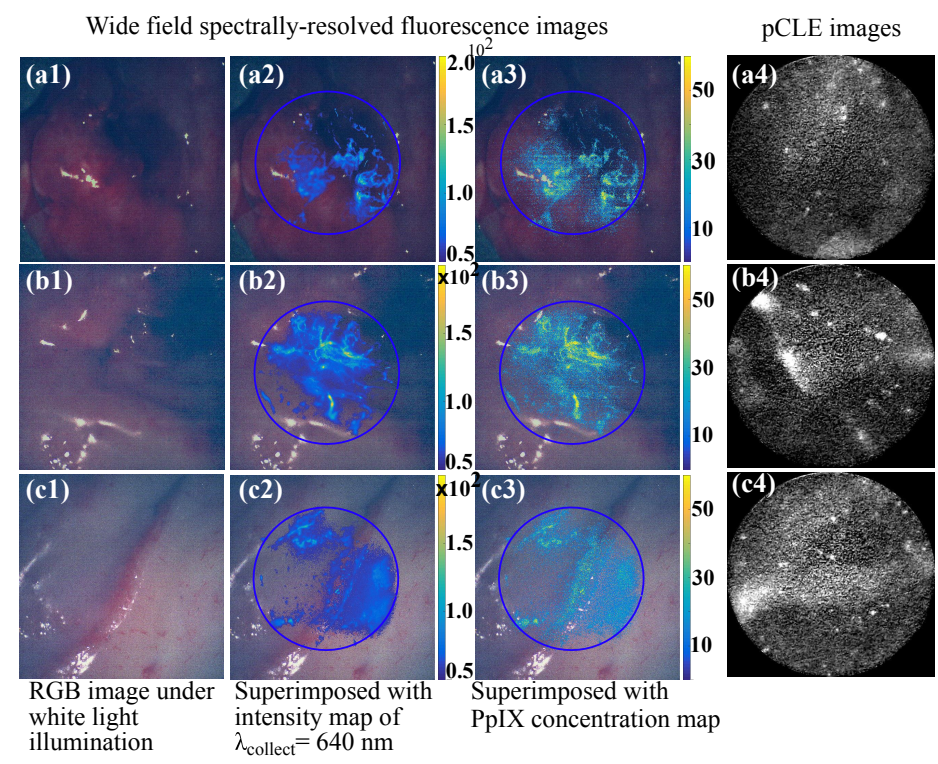

Figure 4. (a1,b1,c1) White light FoV RGB images. (a2,b2,c2) Corresponding fluorescence images demonstrating PpIX fluorescence at $\lambda=640 \mathrm{~nm}$. (a3,b3,c3) Map of estimation of concentration of PpIX displaying $C_{P p I X}>10 \mu g / m l$. $(\mathrm{a} 4, \mathrm{~b} 4, \mathrm{c} 4)$ Fluorescence endomicroscopic images of the corresponding sample area.The blue circles indicate the UVilluminated area. The blue circles indicate the UV-illuminated area.

Ex vivo Human Studies The excised human glioma sample was structured with a large portion of tumour tissue, an infiltrated margin part and a small fraction of normal neocortical tissue as well as some healthy white matter. The consented patient from whom the specimen was obtained was not preoperatively administered with 5-ALA, therefore we manipulated the glioma specimen with topically application of PpIX using the protocol described in the previous section. As shown in Fig.4, spectrally-resolved wide field fluorescence images illustrated the PpIX distribution in the specimen, while endomicroscopic images demonstrated the PpIX-stained anatomic structure in cellular scale. As the topically administration of PpIX was not able to selectively stain on the cancerous cells, the fluorescence signal detected with our system was not correlated with the tumour pathology. Further investigation with 5-ALA involved glioma sample will be carried out to evaluate our system. 


\section{CONCLUSIONS}

In this study, we developed a multi-scale spectrally-resolved quantitative fluorescence imaging modality for better determination of glioma in brain tumour resection. Our proposed system were able to specifically detect the PpIX fluorescent signal and estimate the true concentration of PpIX in brain specimen. We envisage this system will markedly enhance the efficacy of the glioma resection surgery with high specificity.

\section{ACKNOWLEDGMENTS}

This study was funded by the Health Innovation Challenge Fund (HICF-T4-275, WT 97914), a parallel funding partnership between the Department of Health and Wellcome Trust. This study was supported by an Engineering and Physical Sciences Research Council (EPSRC)-funded (EP/P511262/1) UCL Future Leaders award. This work was undertaken at University College London and University College London Hospitals, which received a proportion of funding from the Department of Health's National Institute for Health Research (NIHR), University College London Hospitals Biomedical Research Centre (BRC) funding scheme. The Epilepsy Society Brain and Tissue Bank at University College London is funded by the Epilepsy Society. The views expressed in this publication are those of the author(s) and not necessarily those of the Department of Health or Wellcome Trust.

\section{REFERENCES}

[1] Hardesty, D. A. and Sanai, N., "The value of glioma extent of resection in the modern neurosurgical era," Frontiers in Neurology OCT(October), 1-8 (2012).

[2] Oppenlander, M. E., Wolf, A. B., Snyder, L. A., Bina, R., Wilson, J. R., Coons, S. W., Ashby, L. S., Brachman, D., Nakaji, P., Porter, R. W., Smith, K. A., Spetzler, R. F., and Sanai, N., "An extent of resection threshold for recurrent glioblastoma and its risk for neurological morbidity.," Journal of neurosurgery 120(4), 846-53 (2014).

[3] Schulz, C., Waldeck, S., and Mauer, U. M., "Intraoperative Image Guidance in Neurosurgery: Development, Current Indications, and Future Trends," Radiology Research and Practice 2012, 1-9 (2012).

[4] Ferraro, N., Barbarite, E., Albert, T. R., Berchmans, E., Shah, A. H., Bregy, A., Ivan, M. E., Brown, T., and Komotar, R. J., "The role of 5-aminolevulinic acid in brain tumor surgery: a systematic review," Neurosurgical Review (2016).

[5] Floeth, F. W., Sabel, M., Ewelt, C., Stummer, W., Felsberg, J., Reifenberger, G., Steiger, H. J., Stoffels, G., Coenen, H. H., and Langen, K.-J., "Comparison of 18f-fet pet and 5-ala fluorescence in cerebral gliomas," European Journal of Nuclear Medicine and Molecular Imaging 38(4), 731-741 (2011).

[6] Stummer, W. and Kamp, M. A., "The importance of surgical resection in malignant glioma.," Curr Opin Neurol 22(6), 645-649 (2009).

[7] Puppa, A. D., Ciccarino, P., Lombardi, G., Rolma, G., Cecchin, D., and Rossetto, M., "5-aminolevulinic acid fluorescence in high grade glioma surgery: Surgical outcome, intraoperative findings, and fluorescence patterns," BioMed Research International 2014 (2014).

[8] Teixidor, P., Arráez, M. Á., Villalba, G., Garcia, R., Tardáguila, M., González, J. J., Rimbau, J., Vidal, X., and Montané, E., "Safety and Efficacy of 5-Aminolevulinic Acid for High Grade Glioma in Usual Clinical Practice: A Prospective Cohort Study.," PloS one 11(2), e0149244 (2016).

[9] Haj-Hosseini, N., Richter, J., Andersson-Engels, S., and Wårdell, K., "Optical touch pointer for fluorescence guided glioblastoma resection using 5-aminolevulinic acid," Lasers in Surgery and Medicine 42(1), 9-14 (2010).

[10] Valdés, P. A., Leblond, F., Kim, A., Wilson, B. C., Paulsen, K. D., and Roberts, D. W., "A spectrally constrained dual-band normalization technique for protoporphyrin ix quantification in fluorescence-guided surgery," Optics letters 37, 1817-1819 (06 2012).

[11] Haj-Hosseini, N., Richter, J., Hallbeck, M., and Wårdell, K., "Low dose 5-aminolevulinic acid: Implications in spectroscopic measurements during brain tumor surgery," Photodiagnosis and Photodynamic Therapy 12(2), 209-214 (2015). 
[12] Kim, A., Khurana, M., Moriyama, Y., and Wilson, B. C., "Quantification of in vivo fluorescence decoupled from the effects of tissue optical properties using fiber-optic spectroscopy measurements," Journal of biomedical optics 15(6), 067006 (2010).

[13] Dixon, J. M., Taniguchi, M., and Lindsey, J. S., "Photochemcad 2. a refined program with accompanying spectral databases for photochemical calculations," Photochem. Photobiol. 81(1), 212-213 (2005).

[14] Vercauteren, T., Perchant, A., Pennec, X., and Ayache, N., "Mosaicing of confocal microscopic in vivo soft tissue video sequences," Medical Image Computing and Computer-Assisted Intervention - MICCAI 2005: 8th International Conference, Palm Springs, CA, USA, October 26-29, 2005, Proceedings, Part I, 753-760 (2005).

[15] Gebhart, S. C., Majumder, S. K., and Mahadevan-Jansen, A., "Comparison of spectral variation from spectroscopy to spectral imaging," Appl. Opt. 46, 1343-1360 (Mar 2007).

[16] Valdés, P. a., Leblond, F., Jacobs, V. L., Wilson, B. C., Paulsen, K. D., and Roberts, D. W., "Quantitative, spectrally-resolved intraoperative fluorescence imaging," Scientific reports 2, 798 (2012). 